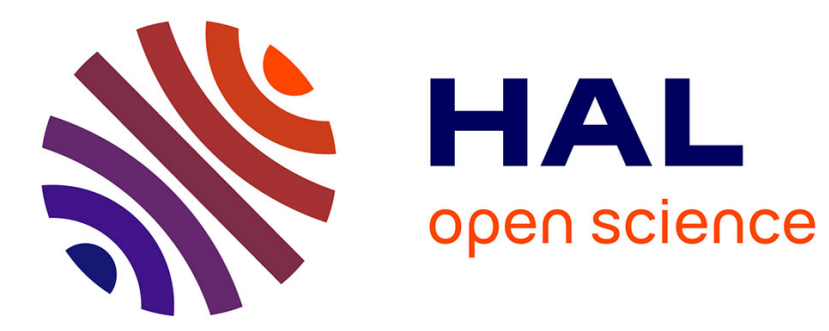

\title{
CALYOU: A Comparable Spoken Algerian Corpus Harvested from YouTube
}

Karima Abidi, Mohamed Amine Menacer, Kamel Smaili

\section{To cite this version:}

Karima Abidi, Mohamed Amine Menacer, Kamel Smaili. CALYOU: A Comparable Spoken Algerian Corpus Harvested from YouTube. 18th Annual Conference of the International Communication Association (Interspeech), Aug 2017, Stockholm, Sweden. hal-01531591

\section{HAL Id: hal-01531591 \\ https://hal.science/hal-01531591}

Submitted on 1 Jun 2017

HAL is a multi-disciplinary open access archive for the deposit and dissemination of scientific research documents, whether they are published or not. The documents may come from teaching and research institutions in France or abroad, or from public or private research centers.
L'archive ouverte pluridisciplinaire HAL, est destinée au dépôt et à la diffusion de documents scientifiques de niveau recherche, publiés ou non, émanant des établissements d'enseignement et de recherche français ou étrangers, des laboratoires publics ou privés. 


\title{
CALYOU: A Comparable Spoken Algerian Corpus Harvested from YouTube
}

\author{
K. Abidi ${ }^{1,2}$, M.A. Menacer ${ }^{2}$ and Kamel Smaïli ${ }^{2}$ \\ ${ }^{1}$ École Supérieure d'Informatique (ESI), Algiers, Algeria \\ ${ }^{2}$ SMarT Group, LORIA, F-54600, France \\ k_abidi@esi.dz, mohamed-amine.menacer@loria.fr, kamel.smaili@loria.fr
}

\begin{abstract}
This paper addresses the issue of comparability of comments extracted from Youtube. The comments concern spoken Algerian that could be either local Arabic, Modern Standard Arabic or French. This diversity of expression gives rise to a huge number of problems concerning the data processing. In this article, several methods of alignment will be proposed and tested. The method which permits to best align is Word2Vecbased approach that will be used iteratively. This recurrent call of Word2 Vec allows us improve significantly the results of comparability. In fact, a dictionary-based approach leads to a Recall of 4, while our approach allows one to get a Recall of 33 at rank 1. Thanks to this approach, we built from Youtube CALYOU, a Comparable Corpus of the spoken Algerian.
\end{abstract}

Index Terms: Algerian dialect, Word2Vec, Comparable corpora, Soundex

\section{Introduction}

Nowadays, to develop speech recognition systems for vernacular languages, is a real challenge for the community. Contrary to classical natural language, vernacular languages are by definition used daily in the communication but are rarely written. To develop a speech recognition system or machine translation for this kind of language, we need to collect data. The best way to get this type of data is to harvest them from social networks. In this work, we are interested by the spoken Algerian. One needs to know that in Algeria people speak their mother tongue, which is a special Arabic dialect, but could speak the official language, which is Modern Standard Arabic (MSA), and also French. Nevertheless, what makes the issue more challenging is that people can mix in the same sentence the three previous languages.

Extracting data from Youtube is not new, Salama et al. in [1] extracted several dialects from this social network in order to constitute a dataset of several dialects. In [2], the authors used Youtube in order to build a Lexicon for the sentiment Analysis. A similar work has been done by [3]. In [4], the authors studied the influence of the sentiment existing in comments of Youtube. Our objective in this work is to collect from Youtube Algerian comments and to align those that are close to each other. To do that, we crawled 22000 videos by using 250 queries related to Algerian topics, such as: Bouteflika, Sellal, Messaoudi, Samira $T v$, Aliouet, etc. Aligning comments will produce a comparable corpus we called CALYOU (Comparable spoken ALgerian extracted from YOUtube). This corpus could be used in several topics such as: adapting a MSA language model to Algerian dialect, to extract parallel dialectal phrases, which may be included in translation tables, etc.

This paper is organized as follows: In Section 2, we present related works concerning the extraction of comparable corpora.
Then, we give an overview on languages spoken in Algeria and their difficulties, in Section 3. We describe the collected corpus in Section 4. Several experiments and results are described in Section 5. Finally, we conclude and present some future works.

\section{Related works}

The comparability topic is widely used in the community and several articles have been published. When documents are collected, one needs to measure the closeness between different articles. The automatic acquisition of comparable corpora can be accomplished by using similarity measures. These measures consist to quantify and capture the different comparability levels. Several related work proposed efficient measures to align and evaluate the quality of extracted comparable documents. Li and Gaussier in [5] defined the degree of comparability between two corpora as the expectation of finding, for each word of the source corpus, its translation in the target one. They use this definition to propose a measure that estimates the comparability of two parallel corpora to which noise have been added. They showed that the comparability degree decreased proportionally with the added noise. A similar approach proposed by Etchegoyhen et al. in [6] termed STACC, is based on expanded lexical sets and Jaccard similarity coefficient. The idea is to get rid of a manual bilingual dictionary. The bilingual dictionary is built on a large parallel corpus by using Giza $++[7]$. Since, it is independent from languages, the approach has been evaluated on a large dataset of ten languages. Huang et al. in [8] describe a method based on techniques inspired from Cross Lingual Information Retrieval. With the translation of the keywords of the source documents, they retrieve the target documents that contain these translated words. Then, the mapping between source and target documents is achieved in accordance to a similarity value. A method based on word embedding has been proposed by Vulic et al in [9]. The model has the possibility to learn bilingual word embeddings from already comparable corpora. The crucial idea in this work is the fact that the method allows to share the cross-lingual embedding space.

Works on comparable corpus containing Arabic are not as popular as those used for English or French, we can mention those proposed in [10],[11], [12]. In this last work, different comparability measures based on bilingual dictionaries or on numerical methods such as Latent Semantic Indexing (LSI) have been proposed.

At the best of our knowledge, there is no work consisting of aligning corpora of the Algerian spoken language in its all forms: writing in MSA, in French and in dialect. Add to that, all the issues related to the way of writing the dialect: Latin script or Arabic script. Also, due to the missing of dictionaries and corpora, no standardization of writing words is available. Concerning resources, except PADIC [13] a multilingual parallel corpus, which contains 6 dialects including two from Algeria, 


\section{The spoken Algerian}

People in Algeria speak mainly Algerian dialect, but this one could be mixed to Modern Standard Arabic and even French. Algerian dialect is informal language, not used in official speech, it includes an important portion of borrowed words, especially from French, Turkish and Berber. Vocabulary of Algerian dialect includes verbs, nouns, pronouns and particles. Most of this vocabulary is from MSA. Moreover, there is significant variation in the vocalization in most cases, and omission of some letters in other cases (mainly the Hamza) ${ }^{1}$. As presented before, in this paper we are interested by the comments of Algerian videos. We have to mention that there is no rule in the way of writing the comments and this constitutes a real challenge for the community. Because no standardization exists, people write sometimes Algerian dialect by using Latin script $(L S)$, called also Arabizi that is an alphabet to communicate over Internet. This obviously constitutes a serious issue because Arabic NLP tools could not be used. Unfortunately, French NLP tools cannot be used either since the written words in $L S$ are not in French. In the following, we give an example of a post related to an Algerian video:

$w$ jma3atkom t3ayeb f bouteflika aw rah khirmanek b alf khatra. That should be written with Arabic script $(A S)$ such as: و .حاعتكم تعيب في بوتفليقة او راه خير منك بالف خطرة and means: And you are making fun of Bouteflika when he is a thousand times better than you.

One can remark that people use some codification for specific Arabic letters that do not exist in $L S$. This is the case of $\varepsilon$ that is written 3 , ق that is written $9, \dot{C}$ as 5 and other codes that are not officially unfortunately adopted by everyone.

Algerian dialect could be written by using $A S$, but some words are not Arabic, they are in general adapted from French such as: بلاصتو that means its place. The sound / p / does not exist in Arabic, but in Algerian dialect it is used as it is and sometimes it is replaced by / b /.

In addition to the Algerian mother tongue, people may express their opinions in French, while some propose their comments in Modern Standard Arabic.

This diversity of the different ways to write comments show the large panel of writing the spoken language that make the processing very challenging.

\section{Corpus}

To build CALYOU, we harvested data from Youtube by using the Google's API ${ }^{2}$ that allows users to search for videos that match specific criteria and retrieve all information and comments of those videos. To harvest, we chose few keywords to form queries in order to retrieve videos concerning national news, Algerian celebrity, football, etc. Table 1 shows some statistics of the collected data, where $|C|$ is the number of comments before and after preprocessing, $|W|$ is the number of words and $|V|$ is the vocabulary size.

We can mention that after the cleaning process, the corpus has been reduced by around $20 \%$ and the vocabulary by around $10 \%$.

\footnotetext{
${ }^{1}$ The Hamza is a letter in the Arabic alphabet, representing the glottal stop.

${ }^{2}$ Available at: https://developers.google.com/Youtube
}

\begin{tabular}{lcc}
\hline & Raw corpus & Cleaned Corpus \\
\hline$|C|$ & $1.1 \mathrm{M}$ & $853 \mathrm{~K}$ \\
$|W|$ & $16 \mathrm{M}$ & $12.7 \mathrm{M}$ \\
$|V|$ & $97 \mathrm{~K}$ & $88 \mathrm{~K}$ \\
\hline
\end{tabular}

\section{Measuring the comparability}

Our objective is to make comparable Algerian dialect, French and Arabic comments to create CALYOU. What we propose, is not only, to produce comparable French and dialect comments, but also to align dialect comments written in LS with those written in AS.

To this end, we need to measure the comparability of comments, that is why we used classical approaches and proposed new ones adapted to the Algerian dialect.

The methods are evaluated on a test corpus composed of comparable comments built by hand. The performance are given in terms of the classical Recall (R@1, R@5 and R@10),well known in Information Retrieval, are given in Table 3 and will be detailed for each approach in the next sections.

\subsection{Dictionary-based method}

In a first experiment, we used a dictionary-based method proposed by Li and Gaussier (LG) [5]. The comparability measure can be defined as the maximum score between a comment written in $L S$ and all the comments written $A S$. We mention that even if the comment is written in $L S$, that does not mean necessarily that it is a French comment. It could be a dialectal comment written in $L S$ as explained in Section 3.

The size and the quality of dictionary may heavily affect the result of comparability measure. For LG, we used the Open Multilingual WordNet $(\mathrm{OMW})^{3}$ that contains 55373 pairs of Arabic and French words. LG method achieves bad results: 4 at Recall 1 (see Table 3), this was expectable since the dictionary is composed by MSA words and not by Algerian Arabic words. In our best of knowledge, there is no available large Algerian - French dictionary, that is why we used the mentioned dictionary because even if the comments are in Arabic dialect, they contain words in MSA.

\subsection{Indexing words by their sounds}

Since dialect is a spoken language, people may write the same word in different manners, while keeping the same pronunciation. That is why, we decided to improve the last method by indexing the words by their pronunciation or more exactly by their representative sounds. For that, we used Soundex [14], a phonetic algorithm for indexing by sound. Words are encoded by taking advantage of their phonetic form. The encoding is done in both comments in $L S$ and $A S$. All the words in the $L S$ that do not exist in the bilingual dictionary are encoded by Soundex. The underlying hypothesis is that we might consider them such as dialectal words or proper names. Then all the words of the comment written in Arabic are encoded by Soundex. If two strings from respectively LS, AS comments have the same code, we can conclude that, one is the transliteration of the other. Soundex proposes to

\footnotetext{
${ }^{3}$ Available at : http://compling.hss.ntu.edu.sg/omw/
} 
replace each letter by the index of a group of characters. Each group is constituted by the graphemes corresponding to the similar class of sounds (see Table 2). We obviously adapted the original corresponding's table in order to match the graphemes of Youtube comments and Arabic sounds. The characters of Group 0, are ignored unless they appear in the first position of a word. Encoding consists in keeping the first character without any change and the following are encoded in accordance to Table 2. Any word will be represented by a letter followed by three digits. For example, encoding the dialect word حومة, will give two codes (Figure 1) corresponding to the possible transliterations: Houma and 7ouma.

Table 2: Corresponding's table for transliteration

\begin{tabular}{|c|c|c|}
\hline English character & Index & Arabic character \\
\hline A E H I O U W Y & 0 & I \\
\hline B P F & 1 & ب ف \\
\hline C S K G J Q X Z & 2 & ك ق غ ص ش س زج ح \\
\hline $\mathrm{DT}$ & 3 & ظ ط ض ذ د ث ت \\
\hline $\mathrm{L}$ & 4 & $J$ \\
\hline $\mathrm{MN}$ & 5 & من ن \\
\hline $\mathrm{R}$ & 6 & ر \\
\hline
\end{tabular}

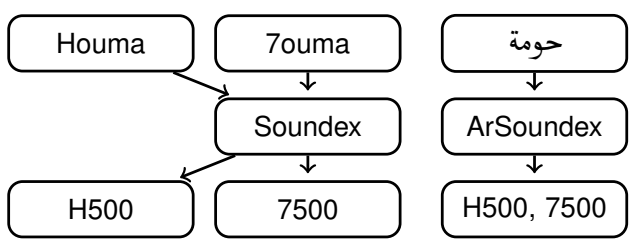

حومة Figure 1: Encoding dialect word

For the previous example حومة will be encoded by " $ح^{500 ", ~}$ then $ح$ is transliterated by 7 or $H$. Consequently, the words , Houma and 7ouma match. Therefore, the process of comparability will provide better results. In fact, we combined LG and Soundex in the process of comparability. The results are given in Table 3 (Line 2). Introducing Soundex allows to improve the results from 4 to 11 at Recall 1 . This is obviously not enough but, henceforth the use of the sound representation of words is necessary.

Table 3: Comparability results

\begin{tabular}{lccc}
\hline & R@1 & R@5 & R@10 \\
\hline$L G$ & 4 & 12 & 19 \\
$L G+$ Sound & 11 & 27 & 40 \\
$S C_{w 2 v}$ & 23 & 42 & 51 \\
$S C_{w 2 v}+$ Sound & 24 & 45 & 52 \\
Iterative SC $C_{w 2 v}+$ Sound & 33 & 48 & 54 \\
\hline
\end{tabular}

\subsection{A Word2Vec-based method}

The free style of writing in the comments of Youtube gives rise to some problems when words should be matched. In fact, the same word in the Algerian spoken could be written in several ways such as in Table 4 . The word kho, which means brother, could be written in various manners in addition to similar words such as hbibna. The word frr is also a way to express the meaning of the word $k h o$; it is derived from the French word frère of which the vowels have been discarded. Because we work on

Table 4: An Example of different ways to write the word kho

\begin{tabular}{lllll}
\hline khoya & khoo & khouya & khou & khyo \\
\hline khooo & hbibna & خويا & frr \\
\hline
\end{tabular}

dialects and there is no dictionary, which could provide all the forms related to one lexical entry, we decided to use Word2Vec to retrieve the different forms of a word. To find the correlated words for each entry of an Algerian lexical dialect, we opted for a continuous bag of words (CBOW) method [15] with a sliding window of 150 . This size has been fixed after several tests. This large number is due to the fact that all the comments concerning the same video have been concatenated into one document.

CBOW allows us to build for each word, a list of its correlated words, the idea is to have for each word all its various forms. Table 5 shows the words that are related to kho with the corresponding distances. We can remark that the majority of those words of Table 4 do exist in the list provided by word2Vec. In addition to these words, others are proposed that will be helpful for the comparability. We used these lists of words provided

Table 5: The closest words of kho in accordance to CBOW method

\begin{tabular}{llllll}
\hline khoya & video & khoo & khouya & rak & khou \\
\hline 0.59 & 0.53 & 0.52 & 0.51 & 0.47 & 0.46 \\
\hline khooo & ta3 & hbibna & خو & frr \\
\hline 0.45 & 0.44 & 0.44 & 0.41 & 0.38 & 0.37 \\
\hline
\end{tabular}

by Word2Vec to better estimate the comparability between two comments. The comparability between a comment in LS with a comment in AS is estimated as follows:

$S C\left(C_{L S}, C_{A S}\right)=\frac{\sum_{w \in C_{L S}} \sigma\left(w, C_{A S}\right)+\sum_{w \in C_{A S}} \sigma\left(w, C_{L S}\right)}{\left|C_{L S}\right|+\left|C_{A S}\right|}$

where $C_{L S}$ (respectively $C_{A S}$ ) is Latin Script (respectively Arabic script) of Algerian video comments. $\sigma$ is a function, which increases the value of the comparability, if any word provided by CBOW does exist in the target comment.

$$
\sigma(w, C)=\left\{\begin{array}{l}
1 \text { if } \exists m / m \in C B O W(w) \wedge m \in C \\
0 \text { else }
\end{array}\right.
$$

The comparability measure $S C_{w 2 v}$ can be defined as the maximum score between a Latin script comment $C_{L S}$ with every comment written in Arabic script $C_{A S}^{i}$ for $1 \leq i \leq N_{C_{A S}}$. Where $N_{C_{A S}}$ is the number of comments written in Arabic script. 


\section{References}

[1] A. Salama, H. Bouamor, B. Mohit, and K. Oflazer, "Youdacc: the youtube dialectal arabic comment corpus," in Proceedings of the Ninth International Conference on Language Resources and Evaluation, LREC 2014, Reykjavik, Iceland, May 26-31, 2014., 2014, pp. 1246-1251.

[2] M. Abdul-Mageed, M. Korayem, and A. YoussefAgha, "”yes we can?": Subjectivity annotation and tagging for the health domain," in Recent Advances in Natural Language Processing, RANLP 2011, 12-14 September, 2011, Hissar, Bulgaria, 2011, pp. 666671.

[3] O. Uryupina, B. Plank, A. Severyn, A. Rotondi, and A. Moschitti, "Sentube: A corpus for sentiment analysis on youtube social media," in Proceedings of the Ninth International Conference on Language Resources and Evaluation, LREC 2014, Reykjavik, Iceland, May 26-31, 2014., 2014, pp. 4244-4249.

[4] S. Siersdorfer, S. Chelaru, W. Nejdl, and J. S. Pedro, "How useful are your comments?: analyzing and predicting youtube comments and comment ratings," in Proceedings of the 19th International Conference on World Wide Web, WWW 2010, Raleigh, North Carolina, USA, April 26-30, 2010, 2010, pp. 891-900.

[5] B. Li and É. Gaussier, "Improving corpus comparability for bilingual lexicon extraction from comparable corpora," in COLING 2010, 23rd International Conference on Computational Linguis tics, Proceedings of the Conference, 23-27 August 2010, Beijing, China, 2010, pp. 644-652.

[6] T. Etchegoyhen and A. Azpeitia, "Set-theoretic alignment for comparable corpora," in Proceedings of the 54th Annual Meeting of the Association for Computational Linguistics, ACL 2016, August 7-12, 2016, Berlin, Germany, Volume 1: Long Papers, 2016.

[7] F. J. Och and H. Ney, "A systematic comparison of various statistical alignment models," Computational Linguistics, vol. 29, no. 1 , pp. 19-51, 2003.

[8] D. Huang, L. Zhao, L. Li, and H. Yu, "Mining large-scale comparable corpora from chinese-english news collections," in Proceedings of the 23rd International Conference on Computational Linguistics: Posters, ser. COLING '10, 2010, pp. 472-480.

[9] I. Vulic and M. Moens, "Monolingual and cross-lingual information retrieval models based on (bilingual) word embeddings," in Proceedings of the 38th International ACM SIGIR Conference on Research and Development in Information Retrieval, Santiago, Chile, August 9-13, 2015, 2015, pp. 363-372.

[10] D. S. Munteanu and D. Marcu, "Improving machine translation performance by exploiting non-parallel corpora," Computational Linguistics, vol. 31, no. 4, pp. 477-504, 2005.

[11] S. Abdul-Rauf and H. Schwenk, "On the use of comparable corpora to improve SMT performance," EACL 2009, 12th Conference of the European Chapter of the Association for Computational Linguistics, Proceedings of the Conference, Athens, Greece, March 30 - April 3, 2009, pp. 16-23, 2009.

[12] M. Saad, D. Langlois, and K. Smaili, "Cross-lingual semantic similarity measure for comparable articles," In Advances in Natural Language Processing - 9th International Conference on NLP PolTAL 2014, Warsaw, Poland, September 17-19, 2014. Proceedings, pages 105-115. Springer International Publishing, 2014.

[13] K. Meftouh, S. Harrat, S. Jamoussi, M. Abbas, and K. Smaïli, "Machine translation experiments on PADIC: A parallel arabic dialect corpus," in Proceedings of the 29th Pacific Asia Conference on Language, Information and Computation, PACLIC 29, Shanghai, China, 2015.

[14] S. U. Aqeel, S. M. Beitzel, E. C. Jensen, D. A. Grossman, and O. Frieder, "On the development of name search techniques for arabic," JASIST, vol. 57, no. 6, pp. 728-739, 2006.
[15] T. Mikolov, K. Chen, G. Corrado, and J. Dean, "Efficient estimation of word representations in vector space," CoRR, vol. abs/1301.3781, 2013.

[16] M. Saad, D. Langlois, and K. Smaïli, "Extracting comparable articles from wikipedia and measuring their comparabilities," Procedia - Social and Behavioral Sciences, vol. 95, pp. 40 - 47, 2013. 